Reprod. Nutr. Dévelop., 1986, 26 (1 A), 53-64.

\title{
Effets de deux substances anabolisantes à action œstrogénique sur le muscle urétral du veau. Etude histochimique et morphométrique
}

\author{
A. F. P. WOUK, J. Y. SAUTET, G. VAN HAVERBEKE, P. CABANIE, J. MORE \\ avec la collaboration technique de Christiane GAUFFENY \\ Laboratoire d'Anatomie pathologique des Animaux domestiques, \\ Ecole Nationale Vétérinaire de Toulouse, \\ 23, Chemin des Capelles, 31076 Toulouse Cédex, France.
}

Summary. Effects of two oestrogenic anabolizers on bull calf urethral muscle. A histochemical and morphological study.

The testing of anabolizer utilization in cattle farming needs quick, sure and cheap detection techniques.

The administration of oestrogen to cattle produces hypertrophy of the prostate, bulbourethral glands and urethral muscle. The aim of this study was to quantify the effect of the association of zeranol (an hormonomimetic drug acting as an oestrogen) and trembolone (a steroidic structure originating from testosterone) on the urethral muscle of bull calves which is easy to sample in the slaughter-house. An histoenzymological and morphometric study of the urethral muscle of 90 bull calves that had been treated showed considerable hypertrophy in transversal sections of the muscle fibers that was increased by more than $90 \%$ in the two main types of striated muscle fiber. The morphological method used is proposed for monitoring the meat of untreated bull calves, requested by a class of consumers.

\section{Introduction.}

L'amélioration des performances en élevage bovin et, plus particulièrement du veau, est en partie due à l'utilisation de produits anabolisants. La F.A.O. et I'O.M.S. définissent les anabolisants comme des substances capables d'améliorer la balance azotée des organismes animaux par une augmentation de l'anabolisme protidique (Ruckebusch, 1981) ; il s'agit donc de véritables agents pharmacologiques capables de stimuler la croissance.

Avant la loi du 16 juillet 1984, deux préparations anabolisantes étaient commercialisées en France : - l'une contient seulement du zéranol, substance hormonomimétique provenant de la fermentation du maïs par une moisissure parasite (Giberella zeae) ; - l'autre l'acétate de trenbolone, dérivé stéroïdique de synthèse de la testostérone était utilisée en association avec le zéranol. 
Les organes réagissant le plus fortement à l'administration d'œestrogènes sont la prostate, les glandes bulbo-urétrales, l'urètre ainsi que les muscles lisses et striés de l'appareil uro-génital mâle (Kroes, 1970 ; Joseph, 1975 ; Kroes et al., 1975 ; Beaufaron, 1977).

Parmi les produits de synthèse à action œstrogénique, le zéranol et surtout le diéthyl-stilbestrol sont les deux anabolisants qui suscitent les plus fortes réactions de la prostate chez les bovins. Le trenbolone inhiberait considérablement les modifications histologiques provoquées sur cet organe par les œestrogènes, en particulier la métaplasie (Kroes et al., 1975).

Juqu'à ce jour, la littérature ne fournissait cependant pas de renseignements quantitatifs concernant la réaction du muscle urétral ( $M$. urethralis) des jeunes bovins soumis à l'action de telles substances. Ce muscle, facile à prélever à l'abattoir, paraissait donc être un support intéressant pour une étude histoenzymologique et morphométrique en vue d'établir des comparaisons chiffrées entre des animaux traités et non traités. Cependant, le manque de renseignements morphologiques et topographiques précis concernant le muscle urétral n'autorisait pas l'obtention de lots de prélèvements suffisamment homogènes pour être comparés, les modifications observées pouvant être liées à des variations de structure suivant les individus ou la localisation des prélèvements. C'est pourquoi, nous avons été amenés, dans une étude préalable, à compléter les données anatomiques et histologiques indispensables à la réalisation de ce travail (Wouk et al., 1985).

Des modifications musculaires morphologiques significatives sur les animaux traités, pourraient ainsi conduire à la mise au point d'une technique simple, révélatrice d'une éventuelle action anabolisante sur une carcasse.

\section{Matériel et méthodes.}

1) Les animaux. - Nous avons utilisé 90 veaux mâles entiers, de race Française Frisonne Pie Noire (FFPN) tous élevés en batterie dans les mêmes conditions dans le but d'obtenir des "veaux de lait».

Ces animaux, en parfaite santé, abattus approximativement à l'âge de 110 jours ont fourni des carcasses pesant en moyenne $100 \mathrm{~kg}$.

\section{2) Les traitements.}

- 25 veaux âgés de 55 jours ont été implantés avec 36 mg de zéranol associés à $140 \mathrm{mg}$ d'acétate de trenbolone (1) ;

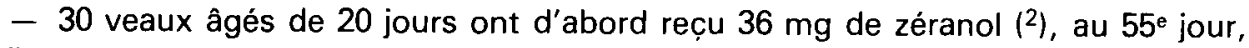
ils ont ensuite reçu une nouvelle dose de $36 \mathrm{mg}$ de zéranol (2) associée à un mélange ( $\left.{ }^{1}\right)$ de $36 \mathrm{mg}$ de zéranol et de $140 \mathrm{mg}$ d'acétate de trenbolone ;

- 35 veaux n'ayant reçu aucun traitement ont été retenus comme témoins.

(1) Forplix (anabolisant protidique, comprimés d'implantation dont la formule est la suivante: - Acétate de trenbolone : $140 \mathrm{mg}$; - Zéranol : $36 \mathrm{mg}$; - Excipient q.s.p. 10 comprimés : 1 dose. (Laboratoire Distrivet).

(2) Ralgro (anabolisant protidique), comprimés d'implantation dont la formule est la suivante : - Zéranol : $36 \mathrm{mg}$; - Excipient q.s.p. 3 comprimés = 1 dose. (Laboratoire Distrivet, $163 \mathrm{av}$. Gambetta, 75020 Paris). 
3) Les méthodes de prélèvements. - Dans les minutes suivant l'abattage, à l'aide d'un bistouri, prélèvement d'un fragment musculaire de $1 \mathrm{~cm}^{3}$, sur la face ventrale de l'urètre et dans le plan médian, selon la technique préconisée par Wouk et al. (1985) (pl. 1).

\section{PLANCHE I}

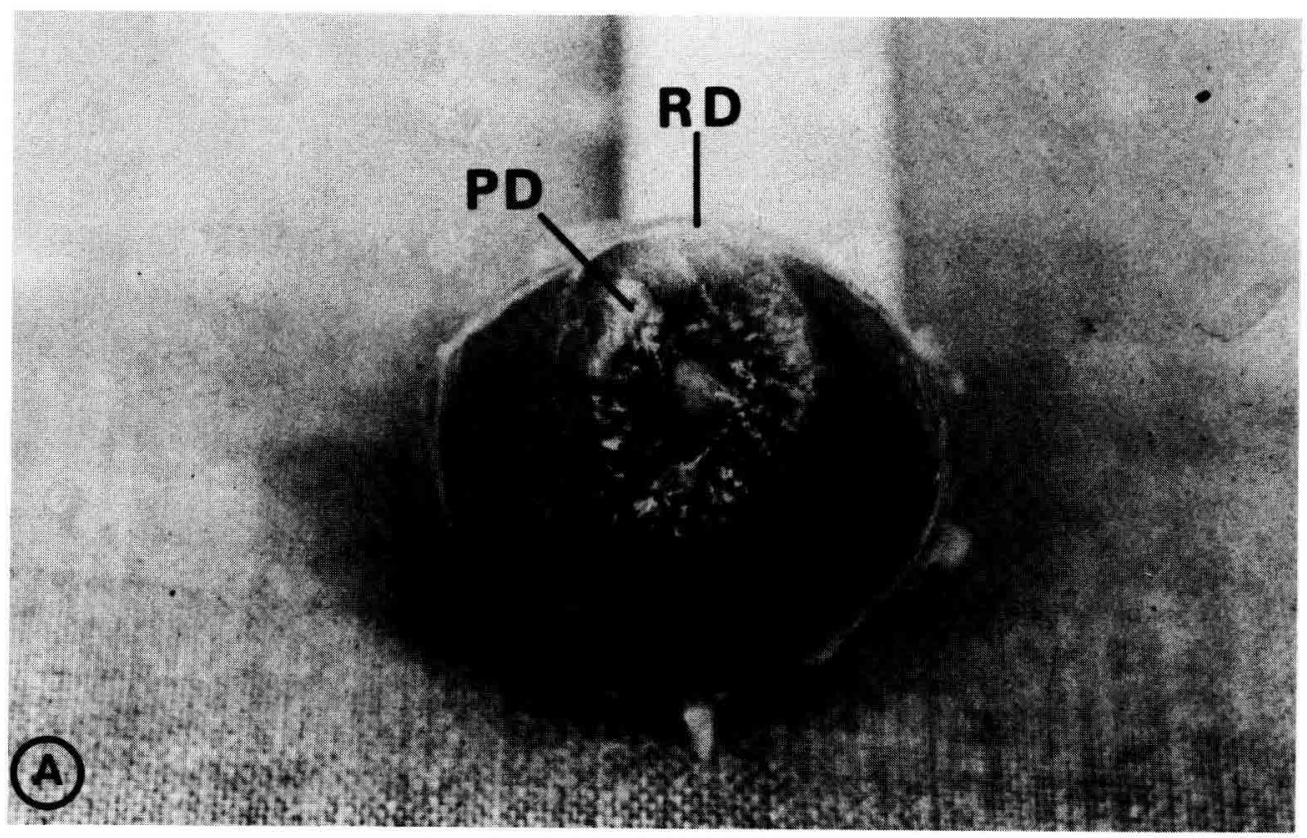

PHOTO A. - Coupe transversale de l'urètre pelvien. Raphé dorsal du Muscle urétral (RD), Prostate disséminée (PD), Muscle urétral : paroi ventrale prélevée (AB), paroi latérale (CD).

L'échantillon est orienté de manière à obtenir des sections transversales des fibres musculaires, puis collé sur une rondelle de liège par une gomme spéciale (Tissue Teck II - O.C.T. Compound 4 fl. Oz. Embedding medium for frozen tissue specimens - Lab. Teck Product).

Le tout est immédiatement fixé par congélation à $-160^{\circ} \mathrm{C}$ grâce à une immersion pendant $10 \mathrm{~s}$ dans de l'isopentane refroidi par de l'azote liquide. Les prélèvements sont ensuite laissés en attente dans un congélateur à $-30^{\circ} \mathrm{C}$.

Réalisation de coupes de $8 \mu$ d'épaisseur à l'aide d'un microtome à congélation à $-20^{\circ} \mathrm{C}$. Les coupes recueillies sur lamelles sont ensuite entreposées pour séchage à $4^{\circ} \mathrm{C}$ pendant $24 \mathrm{~h}$.

4) Techniques de coloration. - Hémalun-éosine ; - Acide périodique de Schiff (P.A.S.) ; - Succinodéshydrogénase (S.D.H.) selon Seligman et Rutemberg (1951) ; - Adénosine triphosphatase (ATPase myofibrillaire) à deux pH de préin- 
cubation différents, $\mathrm{pH} \mathrm{9,4} \mathrm{et} \mathrm{pH} 4,36$; incubation à $\mathrm{pH} \mathrm{9,4,} \mathrm{selon} \mathrm{Brooke} \mathrm{et} \mathrm{Kai-}$ ser (1969).

\section{5) Etude morphométrique.}

Sur 30 prélèvements (témoins, Forplix) : mesure du diamètre de 150 fibres musculaires appartenant à plusieurs faisceaux musculaires, à l'aide d'un oculaire micrométrique $1 / 200^{e}$ après étalonnage avec un micromètre.

Sur 60 prélèvements (témoins, Forplix, association Ralgro + ForplixRalgro) : mesure de surfaces et de pourcentages des différents types de fibres musculaires grâce à un système d'Analyse d'Image Leitz, ASM (Wild. Leitz France, division M.S.I., 86 av. du 18 juin 1940, 92506 Rueil Malmaison Cedex).

La distribution des animaux par traitement reçu et les techniques employées pour chaque type d'étude, sont résumées dans le tableau 1.

\section{TABLEAU}

Distribution des animaux en fonction du traitement reçu, des techniques, méthodes et études réalisées.

\begin{tabular}{|c|c|c|c|c|}
\hline $\begin{array}{l}\text { Nombre } \\
\text { de veaux }\end{array}$ & Traitement reçu & $\begin{array}{l}\text { Fixation, inclusion } \\
\text { colorations } \\
\text { et réactions employées }\end{array}$ & $\begin{array}{l}\text { Méthode } \\
\text { morphométrique }\end{array}$ & Etudes réalisées \\
\hline 15 & Aucun & \multirow{2}{*}{$\begin{array}{l}\text { Coupes à congélation } \\
\text { Hémalun-éosine }\end{array}$} & \multirow{2}{*}{$\begin{array}{l}\text { Oculaire } \\
\text { micrométrique }\end{array}$} & \multirow{2}{*}{$\begin{array}{l}\text { Muscle urétral : } \\
\text { Diamètre des fibres } \\
\text { musculaires }\end{array}$} \\
\hline 15 & Forplix & & & \\
\hline 20 & Aucun & \multirow{3}{*}{$\begin{array}{l}\text { Coupes à congélation } \\
\text { Hémalun-éosine } \\
\text { ATP.ase } \mathrm{pH} 9,4 \\
\text { ATP.ase } \mathrm{pH} 4,36 \\
\text { S.D.H. } \\
\text { P.A.S. }\end{array}$} & \multirow{3}{*}{$\begin{array}{c}\text { Système d'analyse } \\
\text { d'images Leitz } \\
\text { A.S.M. }\end{array}$} & \multirow{3}{*}{$\begin{array}{l}\text { Muscle urétral : } \\
\text { Surfaces inscrites. } \\
\text { Pourcentages } \\
\text { des fibres de type I } \\
\text { et de type II }\end{array}$} \\
\hline 30 & $\begin{array}{l}\text { Ralgro + } \\
\text { Forplix-Ralgro }\end{array}$ & & & \\
\hline 10 & Forplix & & & \\
\hline
\end{tabular}

\section{6) Méthodes statistiques.}

Moyenne arithmétique, écart type et variance ont été calculés à partir des valeurs de chaque prélèvement, ainsi que pour l'ensemble des valeurs du groupe témoin et du groupe traité.

Le test de Cochram a été retenu pour la comparaison des moyennes entre les groupes témoin et traité. Le niveau de signification statistique a été fixé à $5 \%$ pour l'analyse des résultats.

\section{Résultats.}

1) Diamètre des fibres musculaires. - Macroscopiquement on pouvait déjà constater une taille plus importante du muscle urétral chez les animaux traités que chez les animaux témoins. Au microscope, une différence statistiquement signifi- 


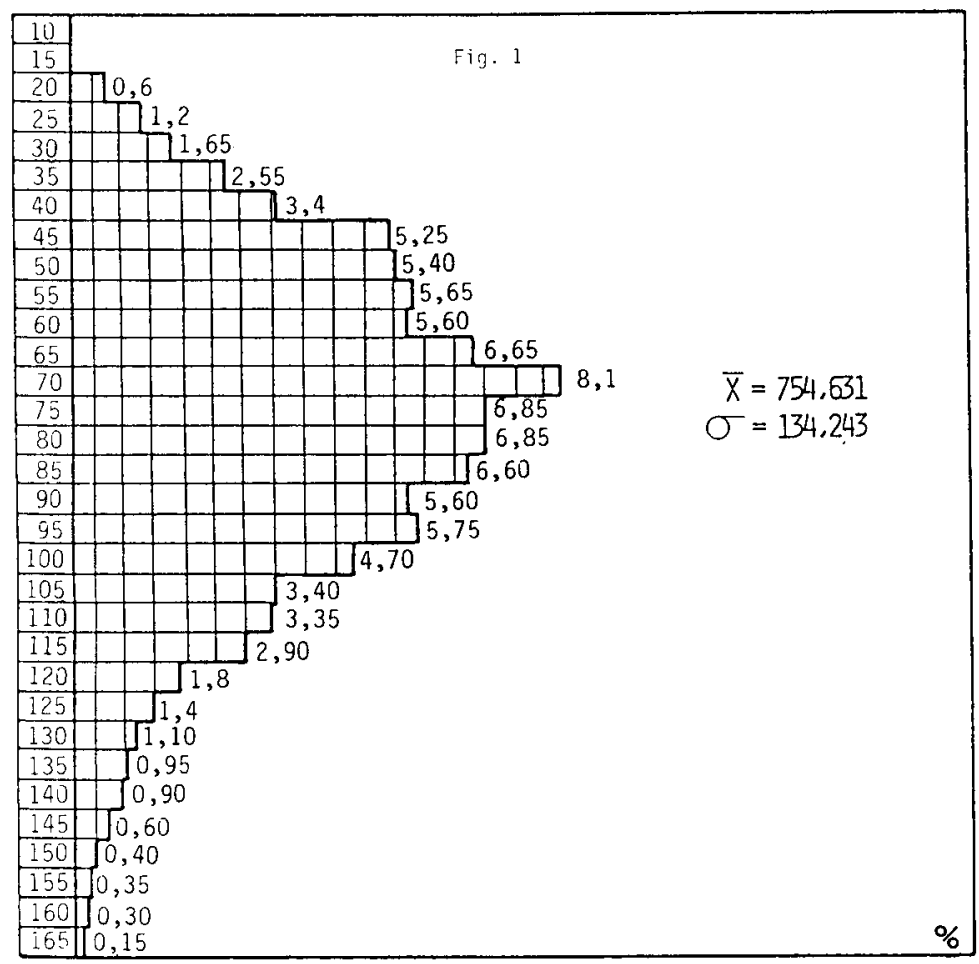

Fig. 1. - Pourcentages de fibres musculaires de type /l par classe de surface en $\mu 2 \times 10-20$ veaux témoins, 2000 fibres mesurées.

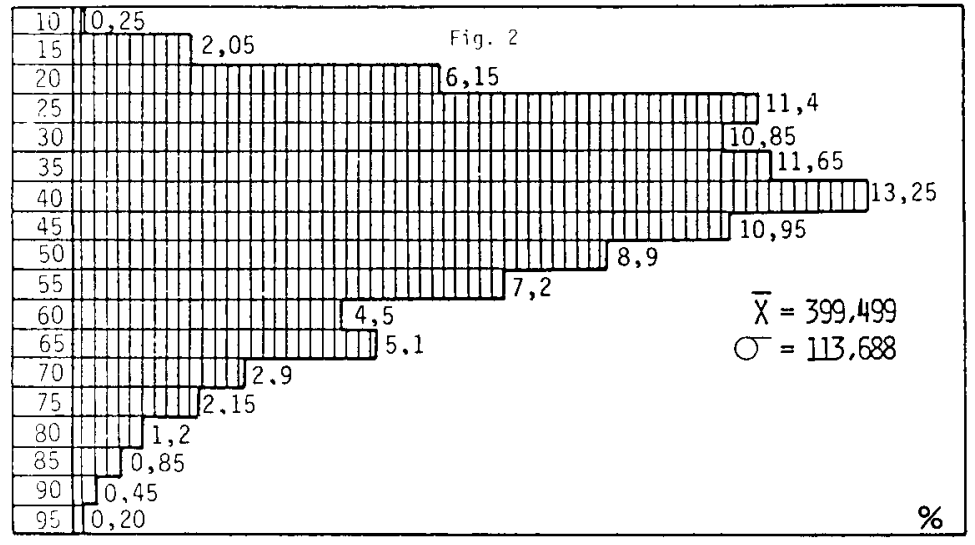

FIG. 2. - Pourcentages de fibres musculaires de type / par classe de surface en $\mu 2 \times 10-20$ veaux témoins, 2000 fibres mesurées. 
cative entre les diamètres moyens des fibres des deux lots est également apparue (tabl. 2).

TABLEAU 2

Diamètre moyen $(\mathrm{x})$ en microns, écart type $(\sigma)$ et variance $\left(\sigma^{2}\right)$ de 2250 fibres de 15 veaux traités au Forplix et de 15 veaux témoins.

\begin{tabular}{lccc}
\hline \multicolumn{1}{c}{ Animaux } & $\mathrm{x}$ & $\sigma$ & $\sigma^{2}$ \\
\hline Témoins & 54,216 & 4,660 & 21,717 \\
\hline Traités & 88,69 & 10,168 & 103,396 \\
\hline
\end{tabular}

2) Surface des fibres musculaires de type / et de type II. - Comme dans le cas précédent, les animaux traités présentaient à l'examen macroscopique des muscles urétraux plus volumineux que les témoins.

L'aspect de toutes les réactions était le même pour les animaux témoins et pour les animaux traités. Aucun signe histologique de dégénérescence musculaire n'a été observé. Au simple examen des coupes, les cellules musculaires des veaux traités présentaient une taille supérieure à celle des veaux témoins.

Les résultats morphométriques des surfaces inscrites sont rassemblés dans le tableau 3 et dans les histogrammes des figures $1,2,3$ et 4 .

\section{TABLEAU 3}

Movenne $(x)$, écart-type $(\sigma)$ et variance $\left(\sigma^{2}\right)$ des surfaces en $\mu^{2}$ des fibres musculaires de type // et de type $/$, en fonction du traitement reçu.

\begin{tabular}{|c|c|c|c|c|c|c|c|}
\hline & $\begin{array}{l}\text { mbre } \\
\text { veaux }\end{array}$ & Traitement reçu & $\begin{array}{c}\text { Type } \\
\text { de fibres }\end{array}$ & $\begin{array}{c}\text { Nombre de fibres } \\
\text { mesurées }\end{array}$ & $x$ & $\sigma$ & $\sigma^{2}$ \\
\hline \multicolumn{2}{|c|}{20} & aucun & II & $\begin{array}{l}2000 \\
2000\end{array}$ & $\begin{array}{l}754,631 \\
399,499\end{array}$ & $\begin{array}{l}134,243 \\
113,688\end{array}$ & $\begin{array}{l}18021,157 \\
12924,666\end{array}$ \\
\hline \multicolumn{2}{|c|}{10} & Forplix & II & $\begin{array}{l}1000 \\
1000\end{array}$ & $\begin{array}{r}1448,097 \\
630,692\end{array}$ & $\begin{array}{l}257,106 \\
111,676\end{array}$ & $\begin{array}{l}66103,379 \\
12471,516\end{array}$ \\
\hline \multicolumn{2}{|c|}{30} & $\begin{array}{c}\text { Ralgro + } \\
\text { Forplix-Ralgro }\end{array}$ & $\begin{array}{c}11 \\
1\end{array}$ & $\begin{array}{l}3000 \\
3000\end{array}$ & $\begin{array}{r}1583,174 \\
705,052\end{array}$ & $\begin{array}{l}292,675 \\
158,583\end{array}$ & $\begin{array}{l}85658,698 \\
25148,470\end{array}$ \\
\hline \multirow{2}{*}{40} & 10 & \multirow{2}{*}{$\begin{array}{c}\text { Forplix } \\
\text { Ralgro }+ \\
\text { Forplix-Ralgro }\end{array}$} & II & 4000 & 1549,05 & 290,157 & 84190,972 \\
\hline & 30 & & I & 4000 & 686,462 & 151,710 & 23015,988 \\
\hline
\end{tabular}

Tant pour les fibres de type I que pour les fibres de type II (pl. II), I'analyse statistique a montré une différence significative entre les groupes suivants: - témoins et ensemble des traités ; - témoins et traités au Forplix ; - témoins et traités au Ralgro + Forplix-Ralgro.

Entre les deux groupes d'animaux traités, pour les deux populations de fibres, il n'a pas été relevé de différences statistiquement significatives. 

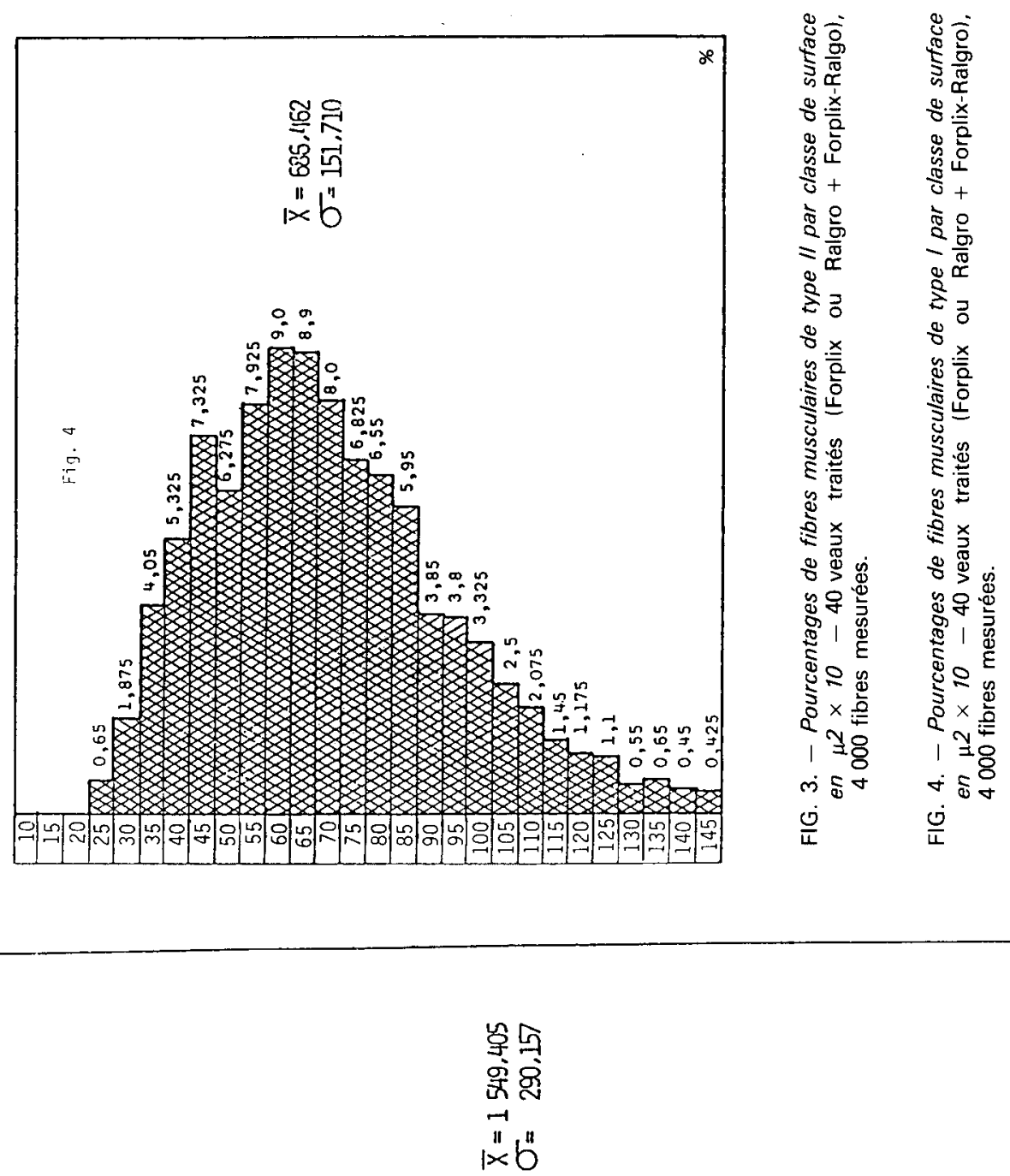

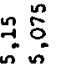

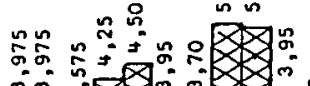

n

N.

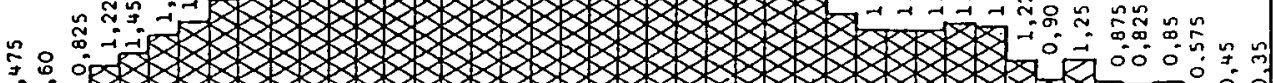

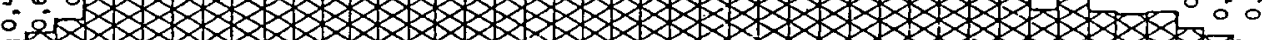

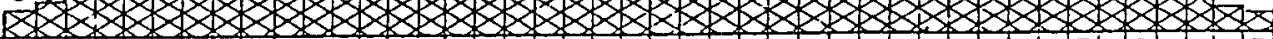
în: 

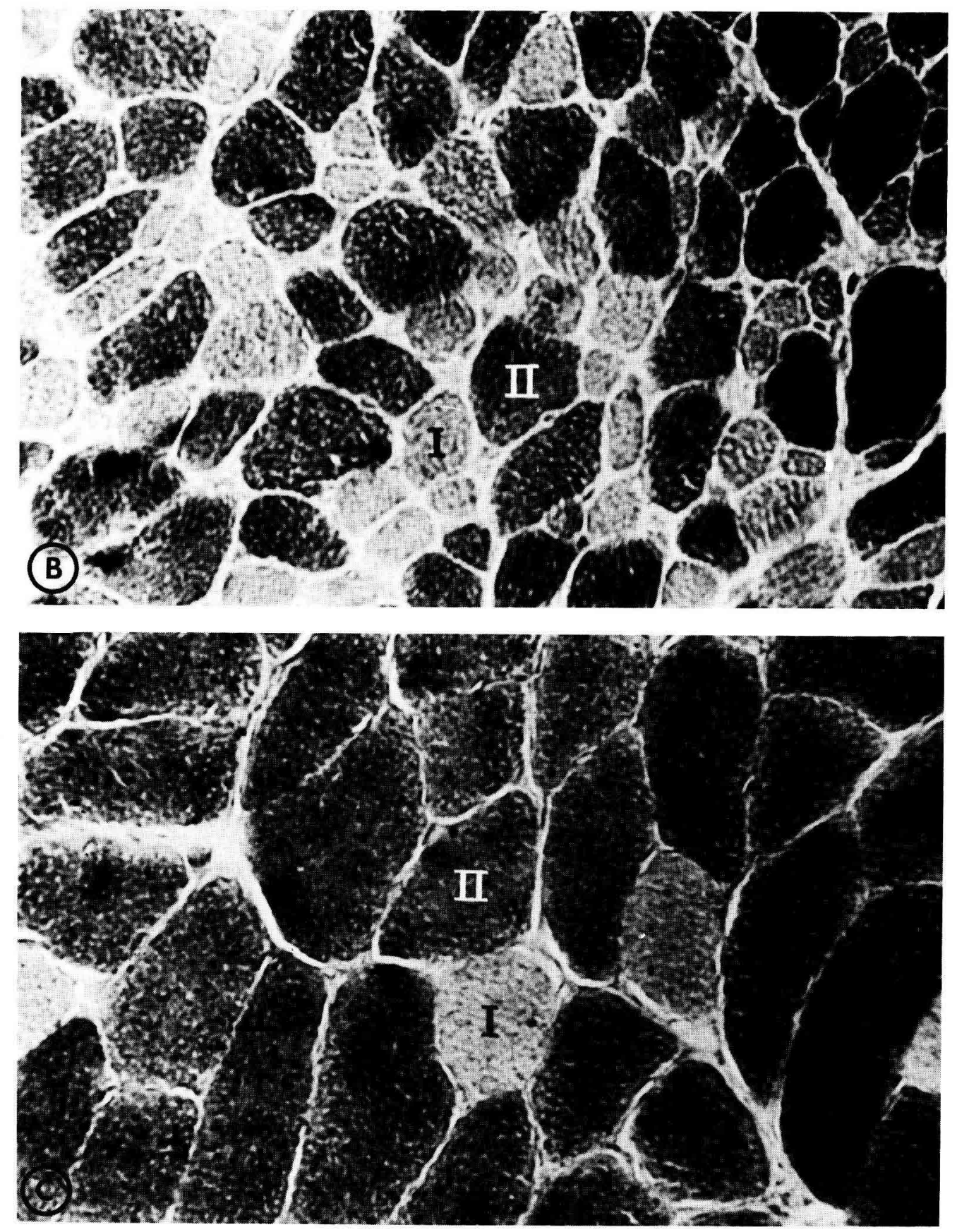
3) Pourcentages des fibres musculaires de type / et de type /I. - Les résultats sont illustrés par les figures 5 et 6 . Par l'application de la méthode de Scheffe, la comparaison des moyennes de pourcentages entre les 3 groupes d'animaux n'a pas montré de différences statistiquement significatives, que le muscle soit prélevé loin du tissu prostatique ou à proximité.

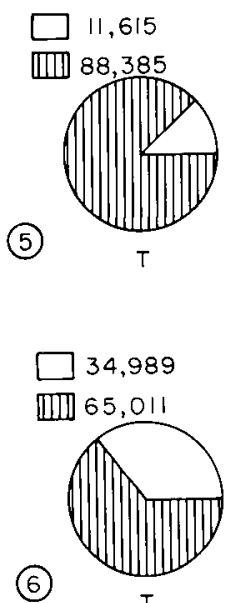

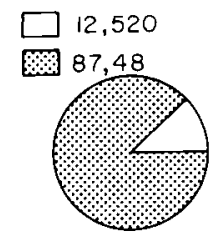

A

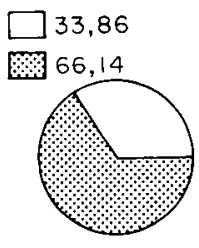

$\Delta$
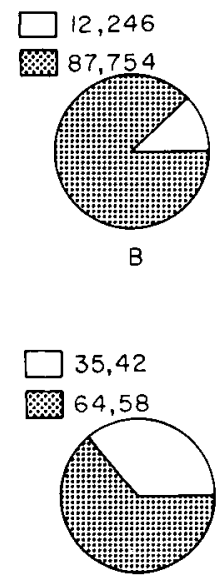

B

FIG. 5. - Pourcentages de fibres musculaires de type / (non tramées) et de type // (tramées) sur 60000 fibres du muscle urétral. Prélèvements effectués à distance de la prostate disséminée sur 60 veaux : - T : 20 veaux témoins : - A : 10 veaux : Forplix ; $-B: 30$ veaux : Ralgro + Forplix-Ralgro.

FIG. 6. - Pourcentages de fibres musculaires de type / (non tramées) et de type // (tramées) sur 20000 fibres du muscle urétral. Prélèvements effectués à proximité de la prostate disséminée sur 20 veaux: - T : 10 veaux témoins; - A : 5 veaux: Forplix; - B : 5 veaux : Ralgro + Forplix-Ralgro.

\section{Discussion et conclusions.}

L'examen critique des résultats précédemment exposés ainsi que la méthodologie employée conduisent à ouvrir deux rubriques.

1) Choix des techniques et méthodes.

a) Le choix de la région médio-ventrale pour le prélèvement du muscle urétral, découle de l'étude anatomique et histologique préalable de l'urètre du veau mâle, réalisée par Wouk et al. (1985). C'est, en effet, dans cette zone que le mus-

\section{PLANCHE II}

PHOTO B. - Coupe transversale de muscle urétral d'un veau témoin. (ATPase à $\mathrm{pH} 9,4 \times 250$ ) I: Fibre de type I; II: Fibre de type II.

PHOTO C. - Coupe transversale de muscle urétral d'un veau traité. (ATPase à pH $9.4 \times 250$ ). I: Fibre de type I; II: Fibre de type II. 
cle est le plus épais et le plus homogène, de par le parallélisme relatif et le diamètre constant de ses fibres musculaires (PI. I).

b) La technique de congélation constitue une phase essentielle de l'ensemble de la procédure. Au vu des quelques difficultés rencontrées, nous ne pouvons que confirmer les recommandations de Ganter et Jolles (1969), en particulier la congélation brusque et rapide à $-160^{\circ}$ de fragments de petite taille. Les échantillons prélevés furent traités avant le quinzième jour de stockage de manière à limiter la diffusion enzymatique.

c) Les réactions choisies, SDH et ATPases, étaient celles préconisées par Holmes et Ashmore (1972) et par Cardinet et Stephens Orvis (1980). La réaction du PAS a été retenue, car selon Lison (1960), elle est la plus spécifique pour la mise en évidence de la fraction glucidique de la cellule, fraction susceptible d'être modifiée par un traitement anabolisant.

d) L'aspect tinctorial de nos réactions histochimiques et histoenzymologiques a été le même que celui décrit par Grau et Walter (1975), Alves (1978), Wheater et al. (1979), Banks (1981), Henry (1981) et Herbison et al. (1982) pour ces mêmes réactions.

e) La mesure des pourcentages respectifs des fibres de types I et II de chaque prélèvement a été effectuée en deux endroits différents par leur degré d'éloignement du tissu prostatique. La raison en est une différence statistiquement significative de la répartition de ces fibres selon leur localisation, la proportion des fibres de type I augmentant en se rapprochant de la prostate disséminée (Wouk et al., 1985).

f) La mesure du diamètre des fibres au lieu de leur surface, n'est que la conséquence de problèmes techniques survenus à l'analyseur d'images. Nous avons préféré exploiter immédiatement les coupes afin de ne pas laisser perdre le bénéfice de l'expérimentation sur 30 animaux dont 15 témoins, catégorie difficile à obtenir d'une manière assurée.

2) Analyse des résultats.

En ce qui concerne l'aspect tinctorial, aucune différence entre les animaux témoins et les animaux traités n'a été observée ; ceci tend à prouver que l'association zéranol-trenbolone, ne provoque pas d'altérations histoenzymatiques perceptibles (SDH et ATPases) et ne modifie pas la teneur glucidique des fibres (PAS).

Le pourcentage respectif des fibres de type I et de type II n'est pas modifié par l'association zéranol-trenbolone, que le muscle prélevé soit proche ou éloigné de la prostate disséminée. Dans ces deux localisations, la majorité des fibres étant de type II, nous pouvons confirmer que le muscle urétral du veau possède un métabolisme à prédominance anaérobie et fonctionne sur un mode de contraction rapide (Wouk et al., 1985).

Le diamètre des fibres des animaux traités au Forplix est supérieur de $60 \%$ à celui mesuré chez les témoins, avec une différence statistiquement significative entre les moyennes $(88,690 \mu$ contre $54,216 \mu$ : tabl. 2$)$. Nous pouvons ainsi affirmer que l'association zéranol-trenbolone, provoque une hypertrophie importante du muscle urétral du veau.

Les surfaces inscrites des fibres des animaux témoins et traités sont différen- 
tes de façon statistiquement significative (tabl. 3), ce qui confirme l'action hypertrophiante de l'association zéranol-trenbolone sur le muscle urétral du veau. Cette action diffère toutefois dans son intensité suivant le type de fibre (tabl. 3), I'hypertrophie étant plus marquée sur les fibres de type II. L'étude des histogrammes montre également des différences : ils présentent un seul pic pour les fibres de type I (fig. 2 et 4), deux pics (fig. 1 et 3) en revanche sont présents pour les fibres de type II, tant chez les témoins que chez les traités. Ce phénomène est vraisemblablement dû à la présence de fibres intermédiaires qui n'ont pas été distinguées des fibres de type II. Par ailleurs, l'écart type et la variance plus élevés pour les fibres de type II que pour les fibres de type I chez les animaux traités, laissent à penser que la réponse des fibres intermédiaires n'est pas exactement superposable à celle des fibres de type II vraies. Cependant, la finalité de ce travail, qui est la recherche d'une méthode simple, révélatrice d'une éventuelle action anabolisante, et non une étude fondamentale sur les réactions du muscle, nous a conduit à ne pas apprécier les sous-populations de type II (IIb, Ilc, Ila) (Brooke et Kaiser, 1970).

Par ailleurs, nous n'avons pas trouvé de différence significative entre les résultats obtenus par les deux protocoles de traitement testés (Forplix et Ralgro + Forplix-Ralgro), le second protocole ne provoquant qu'une augmentation légèrement supérieure au premier (tabl. 3). Nous pouvons en conclure que le fait d'avoir administré $72 \mathrm{mg}$ de Zéranol supplémentaires aux animaux soumis au second traitement, n'a pas provoqué un effet myotrophique très important sur le muscle urétral. Deux hypothèses peuvent expliquer ce résultat :

- manque de trenbolone additionnel, qui normalement, potentialise l'action du zéranol (Benard, 1981);

- saturation des récepteurs des tissus cibles par les anabolisants (Mouthon, 1976 ; Shire, 1976, Document Roussel-Distrivet, 1982).

II faut, cependant, souligner qu'en rassemblant les surfaces inscrites des deux types de fibres dans une seule moyenne, on trouve $577,065 \mu^{2}$ chez les témoins et $1117,756 \mu^{2}$ chez les traités, soit une différence de plus de $93 \%$. II paraît donc possible à l'avenir, chez le mâle, d'envisager une étude morphométrique ne s'appuyant pas sur une étude histoenzymologique préalable.

En outre, I'hypertrophie du muscle urétral, contrairement à celle de la prostate (Kroes et al., 1975), est renforcée par le trenbolone, ce qui désigne tout particulièrement ce muscle chez le veau comme témoin de l'emploi d'anabolisant.

Nous pouvons conclure que l'ensemble des données morphométriques permet de différencier les animaux exempts d'anabolisants, de ceux traités avec l'association zéranol-trenbolone. Au niveau du groupe, l'application est aisée puisqu'aucun des témoins n'a montré la moindre modification musculaire, tandis que tous les animaux ayant un muscle urétral hypertrophié avaient effectivement reçu des anabolisants. Cette différenciation reste cependant imparfaite à l'échelle de l'individu, nos résultats montrant que quelques animaux traités n'ont pas réagi de façon significative.

La méthode morphométrique employée dans cette étude débouche logiquement sur des perspectives d'applications pratiques. Elle pourrait être utilisée comme méthode d'approche dans le contrôle du "veau fermier " (veau "sans hormone ") que certaines associations d'éleveurs produisent en France et cher-

Reproduction Nutrition Développement, $n^{\circ} 1$ A-86. -5 
chent à promouvoir. Cependant, elle semble devoir s'appliquer avec plus de fiabilité à l'identification de lots d'animaux traités plutôt qu'à celle de jeunes bovins pris individuellement. A côté de l'histologie et de l'histochimie de la prostate, des glandes bulbo-urétrales et de la muqueuse de l'urètre, la morphométrie du muscle urétral constituerait un élément nouveau et complémentaire d'autant plus utile que nous avons pu observer un parallélisme des résultats obtenus à partir de ces différents tissus.

Reçu en juin 1985.

Accepté en octobre 1985

\section{Références}

ALVES V., 1978. Etude morphologique et histoenzymologique de quelques muscles en cours de croissance chez les Ovins. Mémoire-Maîtrise es-Sci. vet., Ecole Nationale Vétérinaire de Toulouse. $42 \mathrm{p}$.

BANKS W. J., 1981. Applied veterinary histology. Williams \& Wilkins Ed., Baltimore.

BEAUFARON J. G., 1977. Problèmes hygiéniques et légaux posés par l'emploi des aestrogènes de synthèse et leur dépistage dans les denrées d'origine animale. Th. Doct. vét. Toulouse.

BENARD G., 1981. Utilisation des anabolisants en élevage, 11-15. In Vétérinaire et santé publique, Ecole Nationale Vétérinaire de Toulouse.

BROOKE M. H., KAISER K. K., 1969. Some comments on the histochemical characterization of muscle adenosine triphosphatase. J. Histochem. Cytochem., 17, 431-436.

BROOKE M. H., KAISER K. K., 1970. Muscle fiber type. How many and what kind ? Arch. Neurol., 23, 369-379.

CARDINET III G. H., STEPHENS ORVIS J., 1980. Skeletal muscle function, 545-574. In KANEKO J. J., Clinical biochemistry of domestic animals, 3rd ed., Acad. Press, New York.

GANTER P., JOLLES G., 1969. Histochimie normale et pathologique. Gauthier Villars, Paris.

GRAU H., WALTER P., 1975. Précis d'histologie et d'anatomie microscopique des animaux domestiques. Vigot frères, Paris.

HENRY M., 1981. Sur quelques conditions métaboliques de l'activité musculaire. Prat. vét. Equine, 13, 7-20.

HERBISON G. J., JAWEED M. M., DITUNNO J. F., 1982. Muscle fiber types. Arch. Phys. Med. Rehabil., 63, 227-239.

HOLMES J. H. G., ASHMORE C. R., 1972. A histochemical study of development of muscle fiber type and size in normal and " double muscled " cattle. Growth, 36, 351-372.

JOSEPH R. R., 1975. Les cestrogènes chez les animaux de boucherie et leurs incidences sur la santé publique. Th. Doct. vét. Toulouse.

KROES R., 1970. Estrogen induced changes in the genital tract of the male. Calf-morphological study. Doct. Th., Utrecht.

KROES R., HUIS IN'TVELD L. G., SCHULLER P. L., STEPHANY R. W., 1975. Methods for controlling the application of anabolics in farm animals. Verlag Georg thieme, Stuttgart.

LISON L., 1960. Histochimie et cytochimie animales. Principes et méthodes. Gauthier Villars, Paris.

MOUTHON G., 1976. Mode d'action biochimique des anabolisants. Point vét., 17, 71-74.

ROUSSEL-DISTRIVET, 1982. Anabolisants et production du veau. Suppression volontaire des anabolisants. Laboratoire Distrivet, 163, avenue Gambetta, 75020 Paris.

RUCKEBUSCH Y., 1981. Physiologie, pharmacologie, thérapeutique animales. 2e ed. Maloine, Paris.

SELIGMAN A. M., RUTENBERG A. M., 1951. The histochemical demonstration of succinic deshydrogenase. Science, 113, 317-320.

SHIRE J. G. M., 1976. The forms uses and significance of genetic variation in endocrine systems. Biol. Rev., 51, 105-110.

WHEATER P. R., BURKITT H. G., DANIELS V. G., 1979. Histologie fonctionnelle. Manuel et At/as. Medsi, Paris.

WOUK A. F. P., SAUTET J. Y., CABANIE P., VAN HAVERBEKE G., 1985. Le Muscle urétral (Musculus urethralis) du Veau mâle, données anatomiques, histologiques et morphométriques. Zbl. Vet. Med. C., Anat. Histol. Embryol. (sous presse). 